\title{
ON THE CONVERGENCE OF QUADRATIC VARIATION FOR COMPOUND FRACTIONAL POISSON PROCESSES IN "FCAA" JOURNAL
}

\author{
Enrico Scalas ${ }^{1}$, Noèlia Viles ${ }^{2}$
}

\begin{abstract}
The relationship between quadratic variation for compound renewal processes and $M$-Wright functions is discussed. The convergence of quadratic variation is investigated both as a random variable (for given $t$ ) and as a stochastic process.

Key Words and Phrases: Continuous time random walk; Inverse stable subordinator; Mittag-Leffler waiting time; Fractional Poisson process; MWright functions; Quadratic variation; Compound renewal process.
\end{abstract}

\section{Introduction}

This paper discusses the relationship between quadratic variation for pure jump processes (usually called continuous-time random walks by physicists) and $M$-Wright functions, often appearing in fractional calculus. Some results highlighted in this short paper were already published in [6]. However, the main focus of that paper was defining and studying the properties of stochastic integrals driven by pure jump processes. Moreover, in this paper, we study the convergence of quadratic variation as a stochastic process.

\subsection{Continuous-Time Random Walks}

Let $\left\{J_{i}\right\}_{i=1}^{\infty}$ be a sequence of independent and identically distributed (i.i.d.) positive random variables with the meaning of durations between

(c) Year Diogenes Co., Sofia

pp. $\mathrm{xxx}-\mathrm{xxx}$ 
events in a point process. They define a renewal process. Let

$$
T_{0}=0, \quad T_{n}=\sum_{i=1}^{n} J_{i}, \text { for } n \geqslant 1,
$$

be the epoch of the $n$-th event. Then the process $N_{t}$ counting the number of events up to time $t$ is

$$
N_{t}=\max \left\{n: T_{n} \leq t\right\},
$$

where it is assumed that the number of events occurring up to $t$ is a finite, but arbitrary, non-negative integer.

Let $\left\{Y_{i}\right\}_{i=1}^{\infty}$ be a sequence of i.i.d. random variables (also independent of the $J_{i}$ s) with the meaning of jumps taking place at every epoch; then the compound process $X(t)$ defined by

$$
X(t)=X_{N_{t}}=\sum_{i=1}^{N_{t}} Y_{i}
$$

is called uncoupled continuous-time random walk (CTRW).

Let $F_{Y}(x)=\mathbb{P}(Y \leq x)$ be the cumulative distribution function for the jumps $Y$, then, by purely probabilistic arguments, one can prove that the cumulative distribution function for $X(t)$ is given by

$$
F_{X(t)}(x)=\mathbb{P}(X(t) \leq x)=\sum_{n=0}^{\infty} \mathbb{P}\left(N_{t}=n\right) F_{Y}^{* n}(x)
$$

where $F_{Y}^{* n}(x)$ is the $n$-fold convolution of $F_{Y}(x)$. As an example, if durations are exponentially distributed $(J \sim \exp (\lambda))$ then the counting process is Poisson $\left(N_{t} \sim \operatorname{Poi}(\lambda t)\right)$; moreover, if the jumps are normally distributed, i.e. $Y \sim N(\mu, \sigma)$, equation (1.4) becomes

$$
F_{X(t)}(x)=\mathrm{e}^{-\lambda t} \sum_{n=0}^{\infty} \frac{(\lambda t)^{n}}{n !} \Phi\left(x \mid n \mu, n \sigma^{2}\right),
$$

where

$$
\Phi\left(x \mid n \mu, n \sigma^{2}\right)=\frac{1}{2}\left[1+\operatorname{erf}\left(\frac{x-n \mu}{\sqrt{2 n \sigma^{2}}}\right)\right]
$$

and

$$
\operatorname{erf}(x)=\frac{2}{\sqrt{\pi}} \int_{0}^{x} \mathrm{e}^{-t^{2}} d t
$$

is the error function. 
As a final remark, if $F_{Y}(x)$ has a probability density function $f_{Y}(x)=$ $d F_{Y} / d x$, from equation (1.4) one can write for the density $f_{X(t)}(x, t)$

$$
f_{X(t)}(x, t)=\sum_{n=0}^{\infty} \mathbb{P}\left(N_{t}=n\right) f_{Y}^{* n}(x)
$$

where $f_{Y}^{* n}$ is the $n$-fold convolution of $f_{y}(x)$.

\subsection{Diffusive limit for CTRWs}

Assume that the duration $J$ is rescaled by a positive real parameter $r$ so that the rescaled duration is $J_{r}=r J$ and that the jump $Y$ is rescaled by $h$ getting $Y_{h}=h Y$. Let $X_{r, h}(t)$ denote the rescaled CTRW. Further assume that $J$ has a density function $f_{J_{r}}(t)$ whose Laplace transform has the following behaviour for vanishing $r$

$$
\begin{aligned}
\tilde{f}_{J_{r}}(s) & =\mathbb{E}\left(\mathrm{e}^{-s J_{r}}\right)=\int_{0}^{\infty} \mathrm{e}^{-s u} f_{J_{r}}(u) d u, \\
& =1-c_{1}(r s)^{\beta}+o\left(r^{\beta}\right), r \rightarrow 0, c_{1}>0,
\end{aligned}
$$

where $0<\beta \leq 1$, and that the characteristic function of $Y_{h}$ has the following behaviour for vanishing $h$

$$
\begin{aligned}
\widehat{f}_{Y_{h}}(\kappa) & =\mathbb{E}\left(\mathrm{e}^{i \kappa Y_{h}}\right)=\int_{-\infty}^{\infty} \mathrm{e}^{i \kappa u} f_{Y_{h}}(u) d u \\
& =1-c_{2}(h|\kappa|)^{\alpha}+o\left(h^{\alpha}\right), h \rightarrow 0, c_{1}>0
\end{aligned}
$$

where $0<\alpha \leq 2$. If the scaling parameters satisfy the equation

$$
c_{1} r^{\beta}=c_{2} h^{\alpha},
$$

then, in the limit $r, h \rightarrow 0$, one can prove that $f_{X_{r, h}(t)}(x, t)$ weakly converges to $u(x, t)$ given by

$$
u(x, t)=\frac{1}{t^{\beta / \alpha}} K_{\alpha, \beta}\left(\frac{x}{t^{\beta / \alpha}}\right)
$$

with

$$
K_{\alpha, \beta}(u)=\frac{1}{2 \pi} \int_{-\infty}^{\infty} \mathrm{e}^{-i \kappa u} E_{\beta}\left(-|\kappa|^{\alpha}\right) d \kappa
$$

where $E_{\beta}(z)$ is the one-parameter Mittag-Leffler function defined as

$$
E_{\beta}(z)=\sum_{n=0}^{\infty} \frac{z^{n}}{\Gamma(\beta n+1)}
$$

and coinciding with the exponential function for $\beta=1$. Notice that equation (1.12) is the fundamental solution (or Green function) for the Cauchy 
problem of the space-time fractional diffusion equation in $-\infty<x<+\infty$ and $t \geq 0$ :

$$
\frac{\partial^{\beta}}{\partial t^{\beta}} u(x, t)=\frac{\partial^{\alpha}}{\partial|x|^{\alpha}} u(x, t), \quad 0<\beta \leq 1, \quad 0<\alpha \leq 2,
$$

where $\frac{\partial^{\beta}}{\partial t^{\beta}}$ and $\frac{\partial^{\alpha}}{\partial|x|^{\alpha}}$ denote the fractional Caputo and Riesz derivatives, respectively. For this generalized diffusion equation we refer the interested reader to the exhaustive paper by Mainardi, Luchko and Pagnini [17] where analytical and numerical results have been presented.

The transition from the CTRW to the fractional diffusion equation 1.15 in the diffusive limit has been discussed in several papers by Gorenflo and co-workers, see e.g. [9, 10, 26, 27]. Furthermore, Scalas in [28] has provided a simplified method to derive the probability density $u(x, t)$ in the diffusive limit. Indeed, it is sufficient to assume that the duration distribution is Mittag-Leffler (see [18, [5]) with survival function given by

$$
\bar{F}_{J}(t)=1-F_{J}(t)=E_{\beta}\left(-t^{\beta}\right) .
$$

Then, the Laplace transform of the probability density for the rescaled durations is

$$
\widetilde{f}_{J_{r}}(s)=\frac{1}{1+(r s)^{\beta}}=1-(r s)^{\beta}+o\left(r^{\beta}\right) .
$$

Moreover, if the jumps $Y$ are distributed according to a symmetric Lévy $\alpha$-stable distribution, the characteristic function for the rescaled jumps becomes

$$
\widehat{f}_{Y_{h}}(\kappa)=\mathrm{e}^{-(h|\kappa|)^{\alpha}}=1-(h|\kappa|)^{\alpha}+o\left(h^{\alpha}\right) .
$$

Therefore, these hypotheses are compatible with equations (1.9) and (1.10). Indeed, it turns out that they are more general than expected as discussed in [27]. Further notice, that this class of CTRWs includes the normal compound Poisson process (NCPP) as a particular case, for $\alpha=2$ and $\beta=1$. Now, equation (1.8) becomes (see $[27,28,1]$ )

$$
f_{X(t)}(x, t)=\sum_{n=0}^{\infty} \frac{t^{\beta n}}{n !} E_{\beta}^{(n)}\left(-t^{\beta}\right) f_{Y}^{* n}(x),
$$

where

$$
E_{\beta}^{(n)}(z)=\frac{d^{n}}{d z^{n}} E_{\beta}(z)
$$

The characteristic function of $f_{X(t)}(x, t)$ is

$$
\widehat{f}_{X(t)}(\kappa, t)=\sum_{n=0}^{\infty} \frac{t^{\beta n}}{n !} E_{\beta}^{(n)}\left(-t^{\beta}\right) \widehat{f}_{Y}^{n}(\kappa)=E_{\beta}\left(t^{\beta}\left(\widehat{f}_{Y}(\kappa)-1\right)\right)
$$


and, observing that the $n$-th epoch is rescaled as

$$
T_{n}(r)=r T_{n},
$$

it tuns out that the rescaled CTRW has the following characteristic function

$$
\begin{aligned}
\widehat{f}_{X_{r, h}(t)}(\kappa, t) & =E_{\beta}\left(\frac{t^{\beta}}{r^{\beta}}\left(\widehat{f}_{Y_{h}}(\kappa)-1\right)\right) \\
& =E_{\beta}\left(\frac{t^{\beta}}{r^{\beta}}\left(-(h|\kappa|)^{\alpha}+o\left(h^{\alpha}\right)\right)\right) .
\end{aligned}
$$

Now, if $r, h \rightarrow 0$ with the scaling equation (1.11) satisfied for $c_{1}=c_{2}=1$, one gets from 1.23 .

$$
\widehat{f}_{X_{r, h}(t)}(\kappa, t) \rightarrow \widehat{u}(\kappa, t)=E_{\beta}\left(-t^{\beta}|\kappa|^{\alpha}\right),
$$

which is the characteristic function of $u(x, t)$ defined in equation 1.12). This simplified method will be used to derive the distribution of the quadratic variation for the class of CTRWs described above.

\section{Quadratic Variation}

\subsection{Definition}

In [6], the following stochastic integral was defined on CTRWs

$$
\begin{aligned}
I_{a}(t)= & \\
& \sum_{i=1}^{N_{t}}\left[(1-a) G\left(X\left(T_{i-1}\right)\right)+a G\left(X\left(T_{i}\right)\right)\right]\left(X\left(T_{i}\right)-X\left(T_{i-1}\right)\right),
\end{aligned}
$$

where $G(x)$ is a sufficiently smooth ordinary function and $a \in[0,1]$. The properties of $I_{a}(t)$ were investigated in [6]. In particular, it turns out that

$$
I_{a}(t)=I_{1 / 2}(t)+\left(a-\frac{1}{2}\right)[X, Z](t)
$$

where $Z(t)=G(X(t))$ and the covariation or cross variation of $X(s)$ and $Z(s)$ for $s \in[0, t]$ is given by

$$
[X, Z](t)=\sum_{i=1}^{N_{t}}\left[X\left(T_{i}\right)-X\left(T_{i-1}\right)\right]\left[Z\left(T_{i}\right)-Z\left(T_{i-1}\right)\right] .
$$

Essentially, the definition 2.25 is a simplified version of Bichteler-Dellacherie theory of stochastic integration (see [2]) and, for $a=0$, equation $(2.26)$ is a version of the celebrated Ito formula in the case of pure jump processes. 
Notice that one has $X\left(T_{i}\right)-X\left(T_{i-1}\right)=Y_{i}$. If $G(x)=x$, equation 2.26 becomes

$$
I_{a}(t)=I_{1 / 2}(t)+\left(a-\frac{1}{2}\right)[X](t)
$$

with

$$
I_{1 / 2}(t)=\frac{1}{2} \sum_{i=1}^{N_{t}}\left[X\left(T_{i-1}\right)+X\left(T_{i}\right)\right]\left(X\left(T_{i}\right)-X\left(T_{i-1}\right)\right),
$$

and

$$
[X]^{2}(t)=[X, X](t)=\sum_{i=1}^{N_{t}}\left[X\left(T_{i}\right)-X\left(T_{i-1}\right)\right]^{2}=\sum_{i=1}^{N_{t}} Y_{i}^{2}
$$

is the quadratic variation.

\subsection{Diffusive Limit}

The probability density function for the quadratic variation can be obtained from the density of squared jumps, $f_{Y^{2}}(x)$ which, in its turn, can be obtained from a transformation of $f_{Y}(x)$ :

$$
f_{Y^{2}}(x)=f_{Y}(\sqrt{x}) / \sqrt{x} .
$$

Now, by the same arguments leading to equation (1.8), one gets the following density function for the quadratic variation

$$
f_{[X](t)}(x, t)=\sum_{n=0}^{\infty} \mathbb{P}\left(N_{t}=n\right) f_{Y^{2}}^{* n}(x),
$$

where $x>0$. The characteristic function of the quadratic variation can be written as

$$
\widehat{f}_{[X](t)}(\kappa, t)=\sum_{n=0}^{\infty} \mathbb{P}\left(N_{t}=n\right) \widehat{f}_{Y^{2}}^{n}(\kappa) .
$$

Using the same method as in the previous section, let us assume that $\mathbb{P}\left(N_{t}=n\right)$ is the distribution of the Mittag-Leffler counting process discussed in [27, 18],

$$
\mathbb{P}\left(N_{t}=n\right)=\frac{t^{\beta n}}{n !} E_{\beta}^{(n)}\left(-t^{\beta}\right) .
$$

Then the equation 2.33 becomes (see [28] and the analogous equation (1.21)

$$
\widehat{f}_{[X](t)}(\kappa, t)=E_{\beta}\left(t^{\beta}\left(1-\widehat{f}_{Y^{2}}(\kappa)\right)\right) .
$$


If the jumps $Y$ are distributed according to a Lévy $\alpha$-stable distribution, for $x \rightarrow \infty, f_{Y^{2}}(x) \sim x^{-\alpha / 2-1}$, and the sum of $Y_{i}^{2}$ converges to the positive stable distribution of exponent $\alpha / 2$, whose characteristic function is

$$
\widehat{f}_{Y^{2}}(\kappa)=\mathrm{e}^{\left((-i \kappa)^{\alpha / 2}\right)} .
$$

Inserting this distribution in equation 2.35), rescaling as above and taking the proper diffusive limit, the following characteristic function is recovered for the diffusive limit of the quadratic variation:

$$
\widehat{q}(\kappa, t)=E_{\beta}\left(-(-i \kappa)^{\alpha / 2} t^{\beta}\right) .
$$

Notice that $h$ appears in terms of the type $\left(h^{\alpha / 2}\right)^{2}=h^{\alpha}$ as before. Therefore, the probability density of the quadratic variation in the diffusive limit becomes

$$
q(x, t)=\frac{1}{t^{2 \beta / \alpha}} M_{\alpha, \beta}\left(\frac{x}{t^{2 \beta / \alpha}}\right)
$$

where

$$
M_{\alpha, \beta}(u)=\frac{1}{2 \pi} \int_{0}^{\infty} \mathrm{e}^{-i \kappa u} E_{\beta}\left(-(-i \kappa)^{\alpha / 2}\right) d \kappa
$$

with $u>0$. When $\alpha=2 M_{2, \beta}(u)$ coincides with the right half of the $M$-Wright function ([17, 20]) of real argument that will be discussed in Appendix. When $\alpha=2$ and $\beta=1$ (standard diffusion case), a delta function $q(x, t ; D)=\delta(x-2 t)$ is recovered, corresponding to the quadratic variation of the Bachelier-Wiener process, $[X]^{2}(t)=2 t$.

\section{Functional convergence of quadratic variation}

\subsection{Preliminary results}

As in section 1, $J_{i}$ are i.i.d. nonnegative random variables and we assume that $J_{1}$ belongs to the strict domain of attraction of some stable law with index $\beta \in(0,1)$. That is, there exist positive constants $b_{n}>0$ such that we have the following convergence in distribution of random variables

$$
b_{n} \sum_{i=1}^{n} J_{i} \stackrel{d}{\Rightarrow} D_{\beta},
$$

when $n \rightarrow+\infty$ and where $D_{\beta}>0$ almost surely. The limiting random variable $D_{\beta}$ has $\beta$-stable distribution.

For $t \geqslant 0$, we define $T_{t}:=\sum_{i=1}^{[t]} J_{i}$, where $[t]$ denotes the integer part of $t$. Then, taking into account the above convergence and using Example 11.2.18 of [21] it follows that all finite-dimensional distributions of $\left\{c^{-1 / \beta} T_{c t}\right\}_{t \geqslant 0}$ converge to that of $\left\{D_{\beta}(t)\right\}_{t \geqslant 0}$, as $c \rightarrow+\infty$ :

$$
\left\{c^{-1 / \beta} T_{c t}\right\}_{t \geqslant 0} \stackrel{f . d .}{\Rightarrow}\left\{D_{\beta}(t)\right\}_{t \geqslant 0} .
$$


FiguRE 1. $\beta$-stable subordinator and inverse $\beta$-stable subordinator

The limiting process $\left\{D_{\beta}(t)\right\}_{t \geqslant 0}$ is called the $\beta$-stable subordinator. In the class of strictly $\beta$-stable diffusion processes it is the positive-oriented extreme one. It is càdlàg (continuous from the right, with left-hand limits), nondecreasing and it has stationary increments (see for instance, 25]).

We can define the functional inverse of the process $D_{\beta}(t)$ as

$$
D_{\beta}^{-1}(t)=\inf \left\{x \geqslant 0: D_{\beta}(x)>t\right\} .
$$

It has almost surely continuous non-decreasing sample paths and without stationary and independent increments.

For any integer $n \geqslant 0$ and any $t \geqslant 0$, it can be easily proved that the number of jumps by time $t$ is at least $n$ if and only if the $n$-th jump occurs at or before $t$, therefore the following events coincide:

$$
\left\{T_{n} \leqslant t\right\}=\left\{N_{t} \leqslant n\right\} .
$$

From (3.1) and (3.3), we have the following limit theorem for the counting process $\left\{N_{t}\right\}_{t \geqslant 0}$.

Theorem 3.1 (Theorem 3.6., [21]).

$$
\left\{c^{-1 / \beta} N_{c t}\right\}_{t \geqslant 0} \stackrel{f . d .}{\Rightarrow}\left\{D_{\beta}^{-1}(t)\right\}_{t \geqslant 0}, \quad \text { as } \quad c \rightarrow+\infty .
$$

We are interested in the convergence of stochastic processes in the Skorokhod space. Let $D\left([0,+\infty), \mathbb{R}_{+}\right)$denote the space of càdlàg $\mathbb{R}_{+}$-valued functions on $[0,+\infty)$. We endow this space with the usual $J_{1}$-topology introduced in [29] (for more details, see Appendix II).

As a corollary, by using Stone's Theorem [30, the convergence in the Skorokhod space $D\left([0, \infty), \mathbb{R}_{+}\right)$follows.

Corollary 3.1 (Corollary 3.7., [21]).

$$
\left\{c^{-1 / \beta} N_{c t}\right\}_{t \geqslant 0} \stackrel{J_{1}-t o p}{\Rightarrow}\left\{D_{\beta}^{-1}(t)\right\}_{t \geqslant 0}, \quad \text { as } \quad c \rightarrow+\infty .
$$

Now, we assume that the jumps $Y_{i}$ belong to the strict generalized domain of attraction of some stable law with exponent $\alpha \in(0,2)$, then there exist $a_{n}>0$ such that

$$
a_{n} \sum_{i=1}^{n} Y_{i} \stackrel{d}{\Rightarrow} L_{\alpha}, \quad \text { as } \quad c \rightarrow+\infty
$$


where $L_{\alpha}$ denotes a random variable with symmetric $\alpha$-stable distribution.

From Example 11.2.18 [21], we can extend this convergence to the convergence of the corresponding finite-dimensional distributions for the stochastic process $\sum_{i=1}^{[t]} Y_{i}$.

THEOREM 3.2 ( [21]).

$$
\left\{c^{-1 / \alpha} \sum_{i=1}^{[c t]} Y_{i}\right\}_{t \geqslant 0} \stackrel{f . d .}{\Rightarrow}\left\{L_{\alpha}(t)\right\}_{t \geqslant 0}, \text { when } \quad c \rightarrow+\infty .
$$

The limiting process is a symmetric $\alpha$-stable Lévy process with sample path belonging to $D([0,+\infty), \mathbb{R})$. The following result of convergence in the Skorokhod space $D([0,+\infty), \mathbb{R})$ endowed with the $J_{1}$-topology is proved in [22]:

Corollary 3.2 (Theorem 4.1, [22]).

$$
\left\{c^{-1 / \alpha} \sum_{i=1}^{[c t]} Y_{i}\right\}_{t \geqslant 0} \stackrel{J_{1}-t o p}{\Rightarrow}\left\{L_{\alpha}(t)\right\}_{t \geqslant 0}, \text { when } c \rightarrow+\infty .
$$

To conclude this subsection we will introduce the following Functional Central Limit Theorem (FCLT) proved by Meerschaert and Scheffler in [21] which identifies the limit process as a composition of an $\alpha$-stable Lévy process and the functional inverse of a $\beta$-stable subordinator $D_{\beta}^{-1}(t)$, with $\alpha \in(0,2]$ and $\beta \in(0,1)$.

Theorem 3.3 (Theorem 4.2., 22]). Under the distributional assumptions considered above for the waiting times $J_{i}$ and the jumps $Y_{i}$, we have

$$
\left\{c^{-\beta / \alpha} \sum_{i=1}^{N_{t}} Y_{i}\right\}_{t \geqslant 0} \stackrel{M_{1}-t o p}{\Rightarrow}\left\{L_{\alpha}\left(D_{\beta}^{-1}(t)\right)\right\}_{t \geqslant 0}, \quad \text { when } \quad c \rightarrow+\infty
$$

in the Skorokhod space $D([0,+\infty), \mathbb{R})$ endowed with the $M_{1}$-topology.

\subsection{Functional Central Limit Theorem for the quadratic variation}

In this section we prove a functional limit theorem for the quadratic variation of the CTRW $\{X(t)\}_{t \geqslant 0}$ defined in 2.30) under the distributional 
assumptions considered in the subsection above. The limiting process obtained is a subordination of the positive $\alpha / 2$-stable Lévy process by the process $\left\{D_{\beta}^{-1}\right\}_{t \geqslant 0}$ introduced in 3.2 .

Theorem 3.4. Under the distributional assumptions considered in subsection 3.1 for the waiting times $J_{i}$ and the jumps $Y_{i}$, we have

$$
\left\{\left(\frac{1}{n^{2 / \alpha}} \sum_{i=1}^{[n t]} Y_{i}^{2}, \frac{1}{n^{1 / \beta}} T_{n t}\right)\right\}_{t \geqslant 0} \stackrel{J_{1}-t o p}{\Rightarrow}\left\{\left(L_{\alpha / 2}^{+}(t), D_{\beta}(t)\right)\right\}_{t \geqslant 0}, \quad \text { as } \quad c \rightarrow+\infty
$$

in the Skorokhod space $D\left([0,+\infty), \mathbb{R}_{+} \times \mathbb{R}_{+}\right)$endowed with the $J_{1}$-topology. Moreover, we have also

$$
\sum_{i=1}^{N_{n t}} \frac{Y_{i}^{2}}{n^{2 \beta / \alpha}} \stackrel{M_{1}-t o p}{\Rightarrow} L_{\alpha / 2}^{+}\left(D_{\beta}^{-1}(t)\right), \quad \text { as } \quad c \rightarrow+\infty
$$

in the Skorokhod space $D\left([0,+\infty), \mathbb{R}_{+}\right)$with the $M_{1}$-topology, where $L_{\alpha / 2}^{+}(t)$ denotes an $\alpha / 2$-stable positive Lévy process.

P r o o f. Our proof is inspired by Meerschaert and Scheffler's paper [22. Assuming that the jumps $Y_{i}$ belong to the strict generalized domain of attraction of some stable law with exponent $\alpha \in(0,2)$, we have that $Y_{i}^{2} \geqslant 0$ belong to the strict generalized domain of attraction of some stable law with exponent $\alpha / 2 \in(0,1)$. By Corollary 3.2. we have that

$$
\left\{c^{-2 / \alpha} \sum_{i=1}^{[c t]} Y_{i}^{2}\right\}_{t \geqslant 0} \stackrel{J_{1}-t o p}{\Rightarrow}\left\{L_{\alpha / 2}^{+}(t)\right\}_{t \geqslant 0}, \quad c \rightarrow \infty
$$

in the Skorokhod space $D\left([0,+\infty), \mathbb{R}_{+}\right)$with the $J_{1}$-topology, where $L_{\alpha / 2}^{+}(t)$ is a $\alpha / 2$-stable Lévy process on $\mathbb{R}_{+}$.

It can be assumed, without loss of generality, that all of the sample paths of the two-dimensional process (see for instance, p. 197 of [25])

$$
\left\{\left(\frac{1}{n^{2 / \alpha}} \sum_{i=1}^{[n t]} Y_{i}^{2}, \frac{1}{n^{1 / \beta}} \sum_{i=1}^{[n t]} J_{i}\right)\right\}_{t \geqslant 0}
$$


belong to $D\left([0,+\infty), \mathbb{R}_{+} \times \mathbb{R}_{+}\right)$. Then, it follows by the previous results (3.1) and (3.10) that

$$
\left\{\left(\frac{1}{n^{2 / \alpha}} \sum_{i=1}^{[n t]} Y_{i}^{2}, \frac{1}{n^{1 / \beta}} \sum_{i=1}^{[n t]} J_{i}\right)\right\}_{t \geqslant 0} \stackrel{J_{1}-t o p}{\Rightarrow}\left\{\left(L_{\alpha / 2}^{+}(t), D_{\beta}(t)\right)\right\}_{t \geqslant 0} \text {, as } n \rightarrow+\infty
$$

in $D\left([0,+\infty), \mathbb{R}_{+} \times \mathbb{R}_{+}\right)$endowed with the $J_{1}$-topology.

Now by (3.11) and applying the Continuous Mapping Theorem (see in Appendix II), taking the mapping

$$
\begin{gathered}
\Psi: D\left([0,+\infty), \mathbb{R}_{+}\right) \times D\left([0,+\infty), \mathbb{R}_{+}\right) \\
\rightarrow D\left([0,+\infty), \mathbb{R}_{+}\right) \times D\left([0,+\infty), \mathbb{R}_{+}\right) \\
(x, y) \quad \rightarrow \quad(x, y),
\end{gathered}
$$

we obtain

$$
\left(\left\{\frac{1}{n^{2 / \alpha}} \sum_{i=1}^{[n t]} Y_{i}^{2}\right\}_{t \geqslant 0},\left\{\frac{1}{n^{1 / \beta}} \sum_{i=1}^{[n t]} J_{i}\right\}_{t \geqslant 0}\right) \stackrel{J_{1}-t o p}{\Rightarrow}\left(\left\{L_{\alpha / 2}^{+}(t)\right\}_{t \geqslant 0},\left\{D_{\beta}(t)\right\}_{t \geqslant 0}\right),
$$

when $n \rightarrow+\infty$, in $D\left([0,+\infty), \mathbb{R}_{+} \times \mathbb{R}_{+}\right)$with the $J_{1}$-topology, where as usual the topology on product spaces is the product topology.

For a nondecreasing function $y \in D\left([0,+\infty), \mathbb{R}_{+}\right)$, we can define the inverse as

$$
y^{-1}(t)=\inf \{s \geqslant 0: y(s)>t\} .
$$

Now, we will apply again the Continuous Mapping Theorem to (3.12) and with the mapping

$\Phi: D\left([0,+\infty), \mathbb{R}_{+}\right) \times D\left([0,+\infty), \mathbb{R}_{+}\right) \rightarrow D\left([0,+\infty), \mathbb{R}_{+}\right) \times D\left([0,+\infty), \mathbb{R}_{+}\right)$

$$
(x, y) \quad \rightarrow \quad\left(x, y^{-1}\right) \text {. }
$$

We obtain that

$$
\left(\left\{\frac{1}{n^{2 / \alpha}} \sum_{i=1}^{[n t]} Y_{i}^{2}\right\}_{t \geqslant 0},\left\{\frac{1}{n} N_{n t}\right\}_{t \geqslant 0}\right) \stackrel{M_{1}-t o p}{\Rightarrow}\left(\left\{L_{\alpha / 2}^{+}(t)\right\}_{t \geqslant 0},\left\{D_{\beta}^{-1}(t)\right\}_{t \geqslant 0}\right),
$$

when $n \rightarrow+\infty$, in the Skorokhod space in $D\left([0,+\infty), \mathbb{R}_{+} \times \mathbb{R}_{+}\right)$with the $M_{1}$-topology.

Finally, applying another time the Continuous Mapping Theorem, with the continuous mapping

$\Phi: D\left([0,+\infty), \mathbb{R}_{+}\right) \times D\left([0,+\infty), \mathbb{R}_{+}\right) \rightarrow D\left([0,+\infty), \mathbb{R}_{+}\right) \times D\left([0,+\infty), \mathbb{R}_{+}\right)$

$$
(x, y) \quad \rightarrow \quad x \circ y,
$$


to $(3.13)$, we have that

$$
\left(\left\{\frac{1}{n^{2 / \alpha}} \sum_{i=1}^{[n t]} Y_{i}^{2}\right\}_{t \geqslant 0},\left\{\frac{1}{n} N_{n t}\right\}_{t \geqslant 0}\right) \stackrel{M_{1}-t o p}{\Rightarrow}\left\{L_{\alpha / 2}^{+}\left(D_{\beta}^{-1}(t)\right)\right\}_{t \geqslant 0},
$$

as $n \rightarrow+\infty$ in $D\left([0,+\infty), \mathbb{R}_{+} \times \mathbb{R}_{+}\right)$with the $M_{1}$-topology.

The functional convergence of quadratic variation leads to the following conjecture on the integrals defined in 2.25): it might be possible to prove that, under proper scaling, the integral converges in some sense to a stochastic integral driven by the semimartingale measure $L_{\alpha}\left(D_{\beta}^{-1}(t)\right)$. This will be the object of further research.

\section{Acknowledgements}

E.S. and N.V. are indebted to R. Gorenflo and F. Mainardi for many illuminating discussion on this and related issues. E.S. wishes to thank his co-authors G. Germano, M. Politi and R.L. Schilling with whom he published the paper where the relationship between $M$-Wright functions and quadratic variation was discovered.

This work was partially funded by MIUR Italian grant PRIN 2009 on Finitary and non-Finitary Probabilistic Methods in Economics within the project The Growth of Firms and Countries: Distributional Properties and Economic Determinants.

\section{Appendix I: The $M$-Wright functions}

In this appendix, we summarize the definition and the main properties of $M$-Wright functions. For this purpose let us first recall the general Wright function, so named in honour of E. Maitland Wright the eminent British mathematician, who introduced and investigated this function in a series of notes starting from 1933 in the framework of the theory of partitions, see [32, 33, 34] The function is defined by the series representation, convergent in the whole complex plane,

$$
W_{\lambda, \mu}(z):=\sum_{n=0}^{\infty} \frac{z^{n}}{n ! \Gamma(\lambda n+\mu)}, \quad \lambda>-1, \quad \mu \in \mathbb{C},
$$

so $W_{\lambda, \mu}(z)$ is an entire function. Originally, Wright assumed $\lambda>0$, and, only in 1940, he considered $-1<\lambda<0$, see [35]. We note that in the handbook of the Bateman Project [4, Ch. 18, presumably for a misprint, $\lambda$ is restricted to be non-negative. We distinguish the Wright functions of first kind $(\lambda \geq 0)$ and second kind $(-1<\lambda<0)$. 
In his earliest analysis of the time-fractional diffusion-wave equation [15, 16], Mainardi introduced the special Wright function of the second kind

$$
M_{\nu}(z):=W_{-\nu, 1-\nu}(-z)=\sum_{n=0}^{\infty} \frac{(-z)^{n}}{n ! \Gamma(-\nu n+(1-\nu)}, \quad 0<\nu<1,
$$

Later the relevance of this function was recognized also by other authors in several topics related to fractional diffusion processes, including Lévy stable distributions in probability theory. The function was then worth to be distinguished by the name of $M$-Wright function or also Mainardi function, see e.g. 17, 8, 11, 12, 17, 23. Applications in probability theory and stochastic processes are also found in [19, 20].

For the present purposes hereafter we outline the properties of this function, when the independent variable is real, with respect to its Laplace and Fourier transforms. Denoting by $x$ a real variable, we consider the Laplace transform of the unilateral function $M_{\nu}(x)$ with support the positive semiaxis $\mathbb{R}^{+}$and the Fourier transform of the bilateral function $\frac{1}{2} M_{\nu}(|x|)$ with support in all of $\mathbb{R}$, obtained from the unilateral function as even continuation on the negative real semi-axis.

From Mainardi's book see [15], Appendix F, we get for $0<\nu<1$ the following integral transforms:

Laplace transform, with $\operatorname{Re}[s]>0$ and $x \geq 0$ we have

$$
\mathcal{L}\left[M_{\nu}(x) ; s\right]:=\int_{0}^{\infty} \mathrm{e}^{-s t} E_{\nu}(-s) \mathrm{d} s
$$

with $x, \kappa \in \mathbb{R}$.

For the Fourier transform, with $\kappa \in \mathbb{R}$, we have

$$
\mathcal{F}\left[\frac{1}{2} M_{\nu}(|x|) ; \kappa\right]:=\frac{1}{2} \int_{-\infty}^{+\infty} \mathrm{e}^{ \pm i \kappa x} M_{\nu}(|x|) \mathrm{d} x=E_{2 \nu}\left(-\kappa^{2}\right) .
$$

From the Laplace transform for $s=0$ we derive the normalization

$$
\int_{0}^{\infty} M_{\nu}(x) \mathrm{d} x=E_{\nu}(0)=1
$$

Furthermore for the limiting case $\nu=1$ it is known that $M_{\nu}(x)$ reduces to the Dirac delta generalized function centred in $x=1$, that is

$$
M_{1}(x)=\delta(x-1) .
$$

Now we provide the explicit (integral) representation of the $M$-Wright function by inverting the above transforms. 
From the Laplace transform (A.3) the Bromwich formula provides for $x>0$

$$
M_{\nu}(x)=\frac{1}{2 \pi i} \int_{\sigma-i \infty}^{\sigma+i \infty} \mathrm{e}^{s x} E_{\nu}(-s) \mathrm{d} s, \quad \sigma>0 .
$$

If we assume $s=\mp i \kappa$ with $\kappa \in \mathbb{R}$ and $\sigma=0$ we obtain the representation by a Fourier integral. In fact, for $\sigma=0$ we mean for the Bromwich integral its Cauchy principal value $(V P)$ obtained by isolating the origin $s=0$ (the terminal point of the branch cut on the negative real semi-axis). Then we get for $x \geq 0$,

$$
M_{\nu}(x)=\frac{1}{2 \pi} V P \int_{-\infty}^{+\infty} \mathrm{e}^{\mp i \kappa x} E_{\nu}( \pm i \kappa) \mathrm{d} \kappa
$$

From inverting the Fourier transform (A.4) )we get for $-\infty<x<+\infty$,

$$
M_{\nu}(|x|)=\frac{1}{\pi} V P \int_{-\infty}^{+\infty} \mathrm{e}^{\mp i \kappa x} E_{2 \nu}\left(-\kappa^{2}\right) \mathrm{d} \kappa .
$$

We recognize the consistency between the two equations (A.8) (A.9), thanks to the duplication formula, see Eq. (E.74) in [19],

$$
E_{2 \nu}\left(-\kappa^{2}\right)=\frac{1}{2}\left[E_{\nu}(+i \kappa)+E_{\nu}(-i \kappa)\right],
$$

taking into account

$$
|x|= \begin{cases}+x, & x>0 \\ -x, & x<0\end{cases}
$$

Finally, for reader's convenience, we recall how the $M$ Wright function enters in the the fundamental solution $u(x, t)$ and in the quadratic variation $q(x, t)$ for the time-fractional diffusion process obtained from the main text with $\alpha=2$ and $0<\beta \leq 1$. Based on the results outlined in this appendix we recognize for $-\infty<x<+\infty$ and $t \geq 0$,

and

$$
u(x, t)=\frac{1}{2 t^{\beta / 2}} M_{\beta / 2}\left(|x| / t^{\beta / 2}\right),
$$

$$
q(x, t)=\frac{1}{2 t^{\beta}} M_{\beta}\left(|x| / t^{\beta}\right) .
$$

\section{Appendix II: Skorokhod topologies $\left(J_{1}\right.$ and $\left.M_{1}\right)$ and Continuous Mapping Theorem}

In this appendix we introduce the $J_{1}$ and $M_{1}$ topologies for the Skorokhod space. We also provide the statement of the Continuous Mapping Theorem.

Recall that the Skorokhod space denoted by $D([0, a], \mathbb{R})$ (with $a>0$ ) is the space of real functions $x:[0, a] \rightarrow \mathbb{R}$ that are right-continuous and 
have left-hand limits. The functions belonging to $D([0, a], \mathbb{R})$ are also called càdlàg (an acronym for continu à droite, limite à gauche in French) functions. It is possible to endow the Skorokhod space $D([0, a], \mathbb{R})$ with a topology that makes this set a Polish space and for which convergence of sequences can be characterized as follows. In these spaces we can apply Arzelà-Ascoli's Theorem to characterize compact sets. This is very useful to find criteria of convergence. Skorokhod [29] proposed four metric separable topologies on $D([0, a], \mathbb{R})$, denoted by $J_{1}, J_{2}, M_{1}$ and $M_{2}$.

In this appendix we focus on the $J_{1}$ and $M_{1}$ topology. The $J_{1}$ topology is the finest and the closest to the uniform topology. Kolmogorov showed in [13] that $D([0, a], \mathbb{R})$ with $J_{1}$-topology is complete. The complete metric on $D([0, a], \mathbb{R})$ is given by Billingsley in $[3]$.

Let $\Lambda$ denote the set of increasing homeomorphisms in $[0, a]$. We will say that $x_{n} \in D([0, a], \mathbb{R})$ converges to $x_{0} \in D([0, a], \mathbb{R})$ in $J_{1}$-topology if there exists a sequence of increasing homeomorphisms $\lambda_{n}:[0, a] \rightarrow[0, a]$ such that

$$
\sup _{t \in[0, a]}\left|\lambda_{n}(t)-t\right| \rightarrow 0, \sup _{t \in[0, a]}\left|x_{n}\left(\lambda_{n}(t)\right)-x_{0}(t)\right| \rightarrow 0
$$

as $n \rightarrow \infty$. When $x_{0}$ is continuous, the convergence in $J_{1}$ is equivalent to the uniform convergence.

On the other hand, the sequence $x_{n}(t)$ converges to $x_{0}(t)$ in $M_{1}$-topology if there exist parametric representations $(y(s), t(s))$ of the graph $\Gamma_{x_{0}(t)}$ and $\left(y_{n}(s), t_{n}(s)\right)$ of the graph $\Gamma_{x_{n}(t)}$ such that

$$
\lim _{n \rightarrow \infty} \sup _{s \in[0,1]} R\left[\left(y_{n}(s), t_{n}(s)\right) ;(y(s), t(s))\right]=0
$$

where the metric is

$$
R\left[\left(y_{n}(s), t_{n}(s)\right) ;(y(s), t(s))\right]:=\left|y_{n}-y\right|+\left|t_{n}-t\right| .
$$

To conclude this appendix we introduce the Continuous Mapping Theorem. It plays an important role in establishing Functional Central Limit Theorems involving a random time change.

Continuous Mapping Theorem (Theorem 13.2.4., [31]). Suppose that $\left(x_{n}, y_{n}\right) \rightarrow(x, y)$ in $D\left([0, a], \mathbb{R}^{k}\right) \times D_{\uparrow}^{1}$ (where $D_{\uparrow}^{1}$ is the subset of functions nondecreasing and with $\left.x^{i}(0) \geqslant 0\right)$. If $y$ is continuous and strictly increasing at $t$ whenever $y(t) \in \operatorname{Disc}(x)$ and $x$ is monotone on $[y(t-), y(t)]$ and $y(t-), y(t) \notin \operatorname{Disc}(x)$ whenever $t \in \operatorname{Disc}(y)$, then $x_{n} \circ y_{n} \rightarrow x \circ y$ in $D\left([0, a], \mathbb{R}^{k}\right)$, where the topology throughout is $M_{1}$ or $M_{2}$. 
The mode of convergence in the lemma above does not matter, because

on the space $D_{\uparrow}^{1}$ convergence in the $M_{1}$ and $M_{2}$ topologies coincides with pointwise convergence on a dense subset of $[0, \infty$ ), including 0 (see Corollary 12.5 .1 by $[31])$.

\section{References}

[1] L. Beghin and E. Orshingher, Fractional Poisson processes and related planar random motions, Electr. J. Prob. 14, (2009), 1790-1826.

[2] K. Bichteler, Stochastic integration and $L^{p}$-theory of semimartingales, Ann. Probab. 9, No 1 (1981), 49-89.

[3] P. Billingsley, Convergence of Probability Measures,Springer, New York, (2002).

[4] A. Erdély, W. Magnus, F. Oberhettinger, and F.-G. Tricomi, Higher Transcendental Functions, Vol. 3, McGraw-Hill, New York (1955).

[5] D. Fulger, E. Scalas, and G. Germano, Monte Carlo simulation of uncoupled continuous-time random walks yielding a stochastic solution of the space-time fractional diffusion equation, Phys. Rev. E 77 (2008), 021122/1-7.

[6] G. Germano, M. Politi, E. Scalas, and R.L. Schilling, Stochastic calculus for uncoupled continuous-time random walks, Phys. Rev. E 79 (2009), 066102/1-12.

[7] R. Gorenflo, Yu. Luchko and F. Mainardi, Analytical properties and applications of the Wright function, Fract. Calc. Appl. Anal. 2, No 4 (1999), 383-414. E-print http://arxiv.org/abs/math-ph/0701069

[8] R. Gorenflo, Yu. Luchko and F. Mainardi, Wright functions as scaleinvariant solutions of the diffusion-wave equation, J. Comp. Appl. Math. 118, No 1-2 (2000), 175-191.

[9] R. Gorenflo, F. Mainardi, E. Scalas and M. Raberto Fractional calculus and continuous-time finance III: the diffusion limit, in M. Kohlmann and S. Tang (Editors), Mathematical Finance, Birkhäuser Verlag, Basel-Boston-Berlin (2001), pp. 171-180.

[10] R. Gorenflo and F. Mainardi, Fractional diffusion processes: probability distributions and continuous time random walk, In: G. Rangarajan and M. Ding (Editors), Processes with Long Range Correlations, Springer-Verlag, Berlin (2003), pp. 148-166. [Lecture Notes in Physics, No. 621] E-print http://arxiv.org/abs/0709.3990

[11] V. Kiryakova, The special functions of fractional calculus as generalized fractional calculus operators of some basic functions, Comput. Math. Appl. 59, No 3 (2010a), 1128-1141. 
[12] V. Kiryakova, The multi-index Mittag-Leffler functions as important class of special functions of fractional calculus, Comput. Math. Appl. 59, No 5 (2010b), 1885-1895.

[13] A. N. Kolmogorov, On Skorohod convergence, Theory Probab. Appl., Vol 1, 213-222, (1979).

[14] N. Laskin, Fractional Poisson process, Communications in Nonlinear Science and Numerical Simulation 8 (2003), 201-213.

[15] F. Mainardi, On the initial value problem for the fractional diffusionwave equation, in S. Rionero and T. Ruggeri (Editors), 7th Conference on Waves and Stability in Continuous Media (WASCOM 1993), World Scientific, Singapore (1994), pp. 246-251. [Series on Advances in Mathematics for Applied Sciences, Vol. 23]

[16] F. Mainardi, Fractional relaxation-oscillation and fractional diffusionwave phenomena, Chaos, Solitons and Fractals 7, No 9 (1996), 14611477.

[17] F. Mainardi, Yu. Luchko and G. Pagnini, The fundamental solution of the space-time fractional diffusion equation, Fract. Calc. Appl. Anal. 4, No 2 (2001), 153-192. E-print http://arxiv.org/abs/cond-mat/0702419

[18] F. Mainardi, R. Gorenflo, and E. Scalas, A fractional generalization of the Poisson process, Vietnam J. Math. 32, SI (2004), 53-64. E-print http://arxiv.org/abs/math/0701454

[19] F. Mainardi, Fractional Calculus and Waves in Linear Viscoelasticity, Imperial College Press, London (2010).

[20] F. Mainardi, A. Mura, and G. Pagnini, The $M$-Wright function in timefractional diffusion processes: A tutorial survey, International Journal of Differential Equations 2010, Article ID 104505 (2010), 29 pages. E-print http://arxiv.org/abs/1004.2950

[21] M. M. Meerschaert, H. P. Scheffler, Limit Distributions for Sums of Independent Random Vectors: Heavy Tails in Theory and Practice. Wiley Series in Probability and Statistics (2001).

[22] M. M. Meerschaert, H. P. Scheffler, Limit theorems for continuous-time random walks with infinite mean waiting times. J. Appl. Probab. 41, No. 3 (2004), 623-638.

[23] I. Podlubny, Fractional Differential Equations, Academic Press, San Diego (1999). [Mathematics in Science and Engineering, Vol. 198]

[24] O.N. Repin and A.I. Saichev, Fractional Poisson law, Radiophysics and Quantum Electronics 43 No 9 (2000), 738-741.

[25] K. Sato, Lévy Processes and Infinitely Divisible Distributions. Cambridge University Press, Cambridge (1999). 
[26] E. Scalas, R. Gorenflo, F. Mainardi and M. Raberto, Revisiting the derivation of the fractional diffusion equation, Fractals 11S (2003), 281289. E-print http://arxiv.org/abs/cond-mat/0210166

[27] E. Scalas, R. Gorenflo, and F. Mainardi, Uncoupled continuoustime random walks: Solution and limiting behavior of the master equation, Phys. Rev. E 69 (2004), 011107/1-8. E-print http://arxiv.org/abs/cond-mat/0402657

[28] E. Scalas, The application of continuous-time random walks in finance and economics, Physica A, 362: 225-239, 2006.

[29] A. V. Skorokhod, Limit theorems for stochastic processes. Theor. Probability Appl. 1, (1956),261-290.

[30] C. Stone, Weak convergence of stochastic processes defined on semiinfinite time intervals, Proc. Amer. Math. Soc. 14, (1963), 694-696.

[31] W. Whitt, Stochastic-Process Limits: An Introduction to StochasticProcess Limits and Their Application to Queues. Springer, New York (2002).

[32] E.M. Wright, On the coefficients of power series having exponential singularities, Journal London Math. Soc. 8 (1933), 71-79.

[33] E.M. Wright, The asymptotic expansion of the generalized Bessel function, Proc. London Math. Soc. (Ser. II) 38 (1935a), 257-270.

[34] E.M. Wright, The asymptotic expansion of the generalized hypergeometric function, Journal London Math. Soc. 10 (1935b), 287-293.

[35] E.M. Wright, The generalized Bessel function of order greater than one, Quart. J. Math., Oxford Ser. 11 (1940), 36-48.

${ }^{1}$ Dipartimento di Scienze e Innovazione Tecnologica, Università del Piemonte Orientale, viale T. Michel 11, I-15121 Alessandria - ITALY and BCAM-Basque Center of Applied Mathematics, Alameda de Mazarredo 14, E-48009 Bilbao, Basque Country - SPAIN

e-mail: enrico.scalas@mfn.unipmn.it

${ }^{2}$ BCAM-Basque Center of Applied Mathematics, Alameda de Mazarredo 14, E-48009 Bilbao, Basque Country - SPAIN

e-mail:nviles@bcamath.org 